\title{
Evaluating H.265 Real-Time Video Flooding Quality in Highway V2V Environments
}

\author{
Alvaro Torres*, Pablo Piñol ${ }^{\dagger}$, Carlos T. Calafate*, Juan-Carlos Cano*, Pietro Manzoni* \\ ${ }^{*}$ Department of Computer Engineering \\ Universitat Politècnica de València, Valencia, Spain \\ atcortes@batousay.com, \{calafate,jucano,pmanzoni\}@disca.upv.es \\ ${ }^{\dagger}$ Dpto. de Física y Arquitectura de Computadores \\ Miguel Hernández University, Elche, Spain \\ pablop@umh.es
}

\begin{abstract}
Video transmission over VANETs is an extremely difficult task not only due to the high bandwidth requirements, but also due to typical VANET characteristics such as signal attenuation, packet losses, high relative speeds and fast topology changes.

In future scenarios, vehicles will provide other vehicles with information about accidents or congestion on the road, and in these cases offering visual information can be a really valuable resource for both drivers and traffic authorities. Hence, achieving an efficient transmission is critical to maximize the user-perceived quality. In this paper we evaluate solutions that combine different flooding techniques, and different video codecs to assess the effectiveness of long-distance real-time video streaming. In particular, we will compare the most effective video coding standard available (H.264) with the upcoming $\mathbf{H . 2 6 5}$ codec in terms of both frame loss and PSNR.

Index Terms-Video streaming, V2V, VANET, flooding, h.264/AVC, h.265/HEVC
\end{abstract}

\section{INTRODUCTION}

Vehicular ad-hoc networks (VANETs) are receiving a lot of attention from vehicle manufacturers since they promote smarter, cleaner, and safer vehicles. In a near future it is expected that vehicular networks will be as extended as mobile phones are nowadays. Vehicles will be equipped with different sensors which can provide useful information to other drivers or traffic authorities. One of the most useful information a vehicle can provide is the live video of an accident situation, not only allowing the emergency services to know in advance the exact status of the accident, but also for other vehicles to decide whether to change their current route.

VANETs provide one of the most difficult environments to achieve a good transmission quality since this type of networks involve high relative speeds, which cause short connection times and transmission problems such as the Doppler effect. In addition, bandwidth is typically very limited, thus becoming one of the worst case scenarios for real-time video transmission. With these constraints it is necessary to check whether it is possible to achieve real-time video transmission with current broadcasting techniques, and, if so, evaluate the final quality of the obtained video.

The recent approval of the new H.265 video compression standard [1], which intends to replace the widely used and well-known H.264 standard [2], provides a new opportunity for real-time video transmission in critical contexts. The new standard, which outperforms the old one achieving the same video quality with only $50 \%$ of the bit-rate [3], may be one of the key points in the achievement of real-time video transmission in vehicular networks, so it is also necessary to evaluate the differences between both of them.

The rest of the paper is structured as follows. In section II, we review the state of the art in terms of both flooding in wireless networks and video transmission over VANETs. In section III, details are provided about the scenario characteristics and the intended video transmission mechanism. Afterward, section IV presents the tools adopted to simulate the proposed scenario. Section $\mathrm{V}$ presents the obtained results and finally, in section VI, we summarize the conclusions obtained and discuss some future work.

\section{RELATED WORK}

In the existing literature several flooding mechanisms have been presented, although the majority of them are intended for MANETs.

Due to the nature of the IEEE 802.11 protocol, which provides a contention-based broadcast mechanism, flooding protocols are focused on avoiding the broadcast storm problem. YuChee Tseng et al. [4] presented the most basic algorithms to solve this problem. The same author published [5] an improved version of the same algorithms by adding adaptive conditions to further reduce the broadcast storm problem. More specifically, the authors present the basis of flooding in MANET environments. In [4] they present the basic versions of some flooding mechanisms such as the Counter-Based Scheme, the Distance-Based Scheme or the Location-Based Scheme. Based on these algorithms, in [5] they slightly improve these flooding schemes by adding information about the environment to decide whether to rebroadcast a packet.

Martínez et al. [6] proposed another flooding mechanism that takes into account the particularities of VANETs, following the guidelines of the Distance-Based mechanism; in particular, they tweak it to provide a fast dissemination of accident alerts in urban scenarios by using information such as the town layout to achieve a smarter flooding. For highway 
scenarios the algorithm does not significantly differ from the Distance-Based approach.

The field of video transmission over VANETs has been studied by several authors, although the main purpose of most articles on video transmission is entertainment, so the video is streamed from Road Side Units (RSU) into the vehicular network.

Meng Guo et al. [7] presented several scenarios where the live video streaming between vehicles, and from vehicles to RSU, is both feasible and desirable.

F. Soldo et al. [8] presented the SUV protocol, a distributed solution to disseminate video streams in VANETs, although a special MAC layer is required to support TDMA scheduling, which avoids its implementation on actual IEEE 802.11p devices.

Overall, although the aforementioned works provide a good number of statistics, none of them presents actual video quality results such as PSNR, being unaware of decoding problems such as the interdependence between frames.

A first approach towards a proper simulation environment capable of representing real time video transmission was proposed in [9], where authors present a simulation platform and PSNR results, following the guidelines presented in [10], thereby providing a useful approach on how to simulate a correct video transmission.

In this paper we present a performance evaluation of different real time video flooding methods, evaluating the error resilience in terms of frame loss and PSNR for both the standard H.264 codec and the new H.265 standard. To the best of our knowledge it is the first time that the new H.265 video codec has been evaluated in lossy VANET environments.

\section{OVERVIEW OF THE VIDEO DELIVERY STRATEGY}

Based on the current state of the art, we have implemented two video flooding strategies focusing on some desirable characteristics:

1) Achieve a high percentage of delivered packets.

2) Promote a fast message propagation.

3) Make the implementation feasible in a real environment. To meet these goals, our flooding mechanism implementation is located at the application layer, using UDP as the transport mechanism. This choice avoids the possibility of aborting the transmission of a message when it's scheduled for transmission at lower levels, and also impedes us from obtaining MAClayer statistics, such as the state of the wireless channel or the number of collisions, while processing the incoming messages. However, the implementation does not depend of any tweaked MAC layer, and can be deployed on real existing devices.

The two flooding strategies implemented are the CounterBased strategy and the Distance-Based strategy. Below we provide an overview of them.

\section{A. Counter-Based strategy}

The parameters employed by this algorithm are two. The first one is the number of copies $(C)$ that a node should hear to stop rebroadcasting a message, and the second one is the maximum time (MaxTime) to rebroadcast.

The exact behavior of our implementation is the following:

1) When a new message arrives, initialize the seen counter to 0 . If the received message was heard previously, increment the local seen counter.

2) Wait a random time between 0 and MaxTime.

3) If seen $<C$, rebroadcast the message, go back to 2 and wait for MaxTime. Otherwise (seen $>=C$ ), discard the message.

\section{B. Distance-Based strategy}

Our implementation is based on the Counter-Based strategy, adding a new parameter, the minimum distance (MinDistance) to rebroadcast a message. If the node is furthest than the minimum distance from the origin node or the rebroadcaster node it will wait for a period between 0 and MaxTime. If it is closest than MinDistance it will wait for MaxTime.

For this implementation it is assumed that every vehicle is equipped with GPS, that the messages are marked with the position of the original sender and, if applicable, with the position of the rebroadcasting node.

The exact behavior of our implementation is the following:

1) When a new message arrives initialize the seen counter to 0 . If the received message was heard previously, increment the local seen counter.

2) Obtain the distances to the original sending node (OriginalDistance) and the re-broadcaster node (RebroadcasterDistance).

3) Wait for a time between 0 and MaxTime, where the time is inversely proportional to the minimum of the previously obtained distances.

4) If seen $<C$, rebroadcast the message, go back to point number 2, and wait for MaxTime. Otherwise ( seen $>=C$ ), discard the message.

\section{Simulation EnVironment}

The simulation environment consists of three main components: SUMO [11], OMNeT++ [12], and INET [13].

To achieve a realistic vehicle mobility we employ the well known open-source vehicular traffic simulator SUMO (Simulation of Urban MObility), which runs coupled with the event-driven OMNeT++ simulator. To simulate the wireless environment we use the INET package that provides an implementation of the IEEE 802.11p standard, as well as higher application levels such as TCP and UDP.

The transmission range in the INET framework is not defined as a fixed distance. Instead, it requires tuning different parameters such as the frequency or the level of attenuation with distance. To achieve a proper level of similarity with reality, we employed the parameters proposed by Báguena et al. [14].

Since highways are a very specific scenario due to the high speeds, we have reduced the default SUMO step time from $1 \mathrm{~s}$ to $0.1 \mathrm{~s}$. This provides a greater resemblance with 


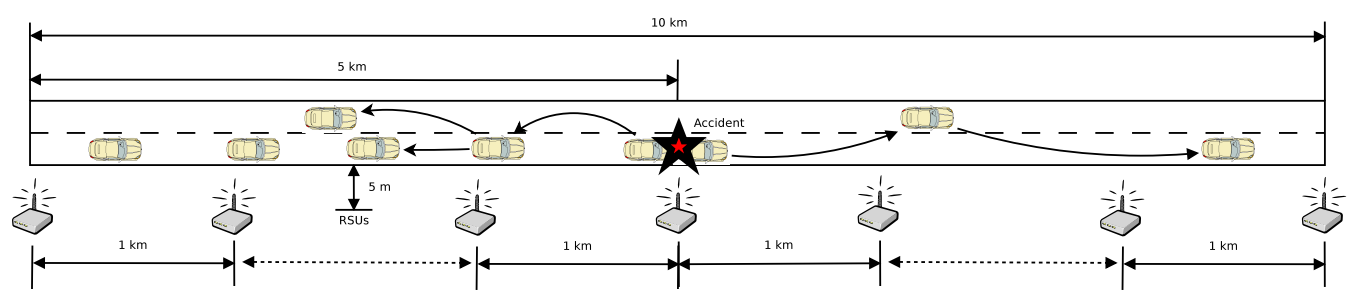

Figure 1. Overview of the simulated scenario.

real-life behavior since vehicles only move for a distance of 3 meters on each mobility simulation step. Another issue handled by SUMO is the speed of the different nodes. We have created four types of vehicles with different probabilities of appearance, and different speeds in order to achieve realistic vehicle behaviors in terms of overtaking, thereby avoiding large vehicle queues on highways.

Concerning the scenario itself, the highway consists of a one-way two-lane $10 \mathrm{~km}$ long straight line. A set of $11 \mathrm{Road}$ Side Units (RSU) have been placed along the road every kilometer. The distance from RSUs to the road is 5 meters. Figure 1 shows our selected scenario.

In our experiments RSUs do not resend any messages, instead, they merely act as traffic sinks. Our goal is to determine the performance of video delivery at RSUs at different distances from the source vehicle.

The vehicle acting as a video source is stopped when it arrives at the middle of the scenario (fifth kilometer) and starts transmitting a video sequence simulating an accident which involves an emergency video stream.

The actual transmitted video is the CIF version of the "Highway" video sequence, which contains 2000 frames [15]. The video has been compressed with the H.264 and H.265 reference encoders both set to a quality level of about $37.9 \mathrm{~dB}$. (37.95 $\mathrm{dB}$ and $37.87 \mathrm{~dB}$ respectively). This quality level corresponds to data rates of $347 \mathrm{kbit} / \mathrm{s}$ and $283 \mathrm{kbit} / \mathrm{s}$ for H.264 and H.265, respectively. Both encoders were configured to create RTP packetized streams, so they have the same overhead. To avoid large dependencies between frames and try to achieve a better packet loss resilience, key-frames are generated every two seconds.

Additionally, to add real-time constraints, we suppose a video buffer of $1 s$, meaning that a vehicle will start the video playback $1 s$ after the arrival of the first packet, and will discard every packet received beyond this jitter threshold.

\section{RESULTS}

This section is structured as follows. First we make an analysis of the different parameters for both flooding algorithms, distance and counter-based, at different vehicle densities. After that, and focusing on the video codecs, we will compare the two codecs analyzed in terms of PSNR and frame loss using the best transmission algorithm according to the previous analysis.

Every single simulation was repeated 25 times, so the points shown in the figures are the mean of the obtained values.

\section{A. Tuning the flooding algorithms}

The main objective of a good flooding algorithm is to reduce the number of transmissions to avoid the broadcast storm problem, while achieving a good packet delivery ratio.

Concerning the first algorithm (Counter-Based) we tested three levels for each parameter, achieving a total of nine configurations. In this case, the $C$ value denotes the additional number of times that a host should hear the message to stop rebroadcasting it, and MaxTime stands for the maximum time (in $\mathrm{ms}$ ) that a node waits when attempting to rebroadcast a message. The second algorithm (Distance-Based) adds another parameter, MinDistance. As explained before, if the receiver node is closer than MinDistance to the sender node, it will wait for MaxTime to rebroadcast the message. If the distance is higher, the node will rebroadcast earlier when the distance to the sender node is higher, to maximize the useful transmissions.

The actual set of values employed for these simulations is the following:

- $C=\{1,2,3\}$

- MaxTime $=\{50 \mathrm{~ms}, 200 \mathrm{~ms}, 333 \mathrm{~ms}\}$

- MinDistance $=\{50 \mathrm{~m}, 250 \mathrm{~m}, 400 \mathrm{~m}\}$

Figure 2 shows the performance of both algorithms when the vehicle inter-arrival time follows an exponential distribution with a mean of $1 s$ (high-density scenario).

The best configuration for the Counter-based solution is, without any doubt, the one with $C=1$ and MaxTime = $333 \mathrm{~ms}$. This allows achieving a mean delivery rate of $72.23 \%$ at the RSUs located five kilometers away, and of $82.63 \%$ at the ones located three kilometers away. This configuration clearly outperforms the other ones by lowering the number of transmissions required. Increasing the number of copies a node should receive to stop rebroadcasting significantly reduces the effectiveness of the flooding process since it just adds more interference to the medium. The same happens when we reduce the MaxTime parameter, as nodes have more probability to collide when gaining access to the medium.

Looking at the right part of figure 2, we can see the results for the Distance-based algorithm. Since the number of different configurations for this algorithm is too high to be shown in a graph, we selected the nine best performing configurations. The best arrival rate at five kilometers distance is obtained by the configuration: $C=1$, MinDistance $=50$ and MaxTime $=200 \mathrm{~ms}$, achieving a packet delivery ratio of $78.5 \%$ at five kilometers, and of $86.45 \%$ at the third kilometer.

If we compare both solutions, the Distance-Based one is able to deliver more packets since it provides a better diffusion 

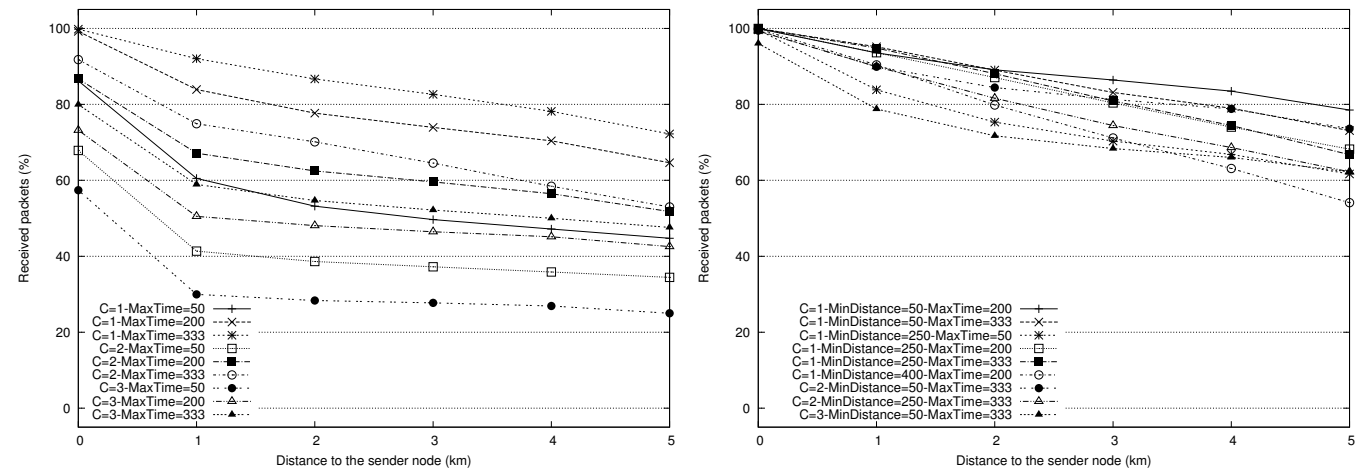

Figure 2. Counter-Based (left) vs. Distance-Based (right). Vehicle arrival rate of 1s.

of the information, prioritizing the furthest nodes and making each transmission more profitable.

Figures 3 and 4, which provide the data for vehicle interarrival times of $2 s$ (medium-density scenario) and $3 s$ (lowdensity scenario), show that, for both densities, the best Counter-Based configuration is the same than in a high-density scenario but, due to the lower number of vehicles, the arrival rate is clearly reduced, achieving only a $50.34 \%$ of delivered packets at a five kilometers distance in the medium-density scenario, and a $38.11 \%$ in the low-density scenario.

Taking into account the Distance-Based algorithms, the right part of figures 3 and 4, show that the previous DistanceBased winner configuration is not the best one for mediumdensity and low-density scenarios. The best configuration for these scenarios is $C=2$, MinDistance $=50$, and MaxTime $=333 \mathrm{~ms}$, achieving a delivery rate at the fifth kilometer of $66.53 \%$ and $46.32 \%$ for medium-density and lowdensity scenarios, respectively. Such differences in terms of optimal configuration are due to collisions near the source vehicle. On the right hand side of figure 3 , focusing on the delivery rates from $0 \mathrm{~km}$ to $3 \mathrm{~km}$, the best configuration is $C=1$, MinDistance $=50$, and MaxTime $=200 \mathrm{~ms}$, while from the third kilometer to the fifth, the best configuration is the one referred above. The main difference between these two configurations is the $C$ parameter as it stands for the number of times a message should be heard before stopping its rebroadcasting.

\section{B. Evaluating the best codec / flooding algorithm combination}

With the purpose of evaluating the actual received video quality we have selected the three best flooding configurations analyzed in previous section.

Figure 5 shows the ratio of packets received for different vehicle arrival rates depending on the selected codec. The three graphs clearly show the differences between flooding backwards or forwards. When flooding backwards (from kilometer 0 to 5 ) the delivery ratio increases as the vehicles start creating a traffic jam, provoking a higher density of vehicles which allows using more vehicles as relays. The opposite process (forward flooding) is a harder task since, when vehicles surpass the accident position, they continue driving at a high speed, therefore creating several disjoint groups.
Focusing on the differences between codecs, when employing the same flooding configuration, the H.264 compressed video has a lower packet arrival rate than the H.265 one due to the higher bitrate that H.264 injects into the network.

The best flooding configuration is different for each codec as they have different traffic demands. The best configuration for the H.264 codec is the Distance-Based algorithm with a configuration of $C=1$, MinDistance $=50 \mathrm{~m}$, and MaxTime $=200 \mathrm{~ms}$. The H.265 codec however, has a lower requirement in terms of bandwidth and allows increasing the $C$ value to add more redundancy without increasing the number of collisions. Figure 5 shows that a DistanceBased flooding algorithm with a configuration of $C=2$, MinDistance $=50 \mathrm{~m}$, and MaxTime $=333 \mathrm{~ms}$ is able to achieve the best packet arrival rate in medium and low-density scenarios while maintaining a good performance in the highdensity scenario.

\section{Error resilience and impact on the video quality}

As in previous section, we have selected the optimum flooding configuration for each codec to show the frame loss as well as PSNR results.

Figure 6 shows the frame loss when employing the best configuration for each codec. While the H.265 codec is able to maintain frame loss at low levels, the H.264 codec suffers an extremely high frame loss when packet losses occur. In fact, the H.264 decoder is often unable to decode several consecutive frames. Although the packet loss difference for both codecs is not very high, H.264 suffers from packet losses in almost every frame, being the decoder unable to properly reconstruct the original video despite using error recovery techniques like frame-freezing. On the other hand, the H.265 decoder is able to compensate for packet losses with the frame-freezing technique, being able to provide a high amount decoded frames, even though they may have some glitches that affect the PSNR and the visual quality.

When the vehicle arrival rate equals to 1s (high-density scenario) the H.264 codec is able to decode all the frames of the video when flooding backwards, while in the same scenario, when flooding forward (from kilometer 5 to 10), significant frame losses occur even for similar packet loss values. To gain further insight into this phenomenon, we have 

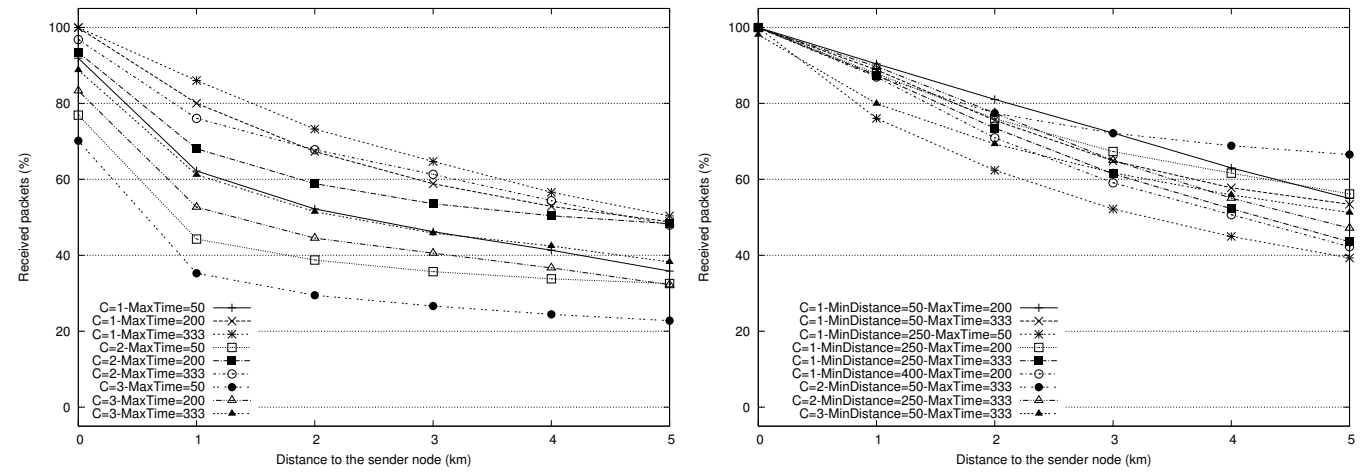

Figure 3. Counter-Based (left) vs. Distance-Based (right). Vehicle arrival rate of $2 \mathrm{~s}$.
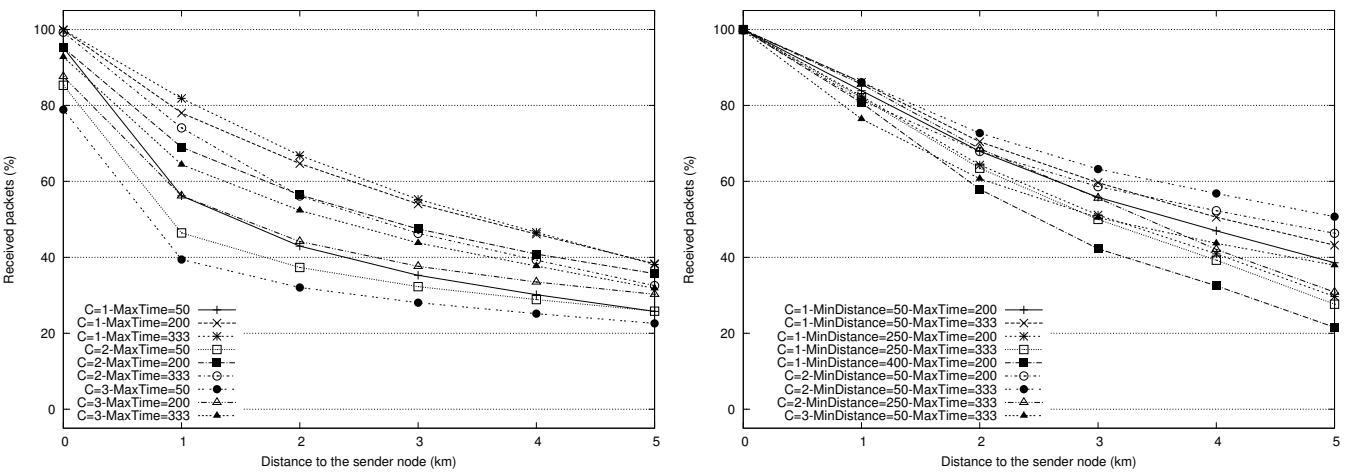

Figure 4. Counter-Based (left) vs. Distance-Based (right). Vehicle arrival rate of 3s.
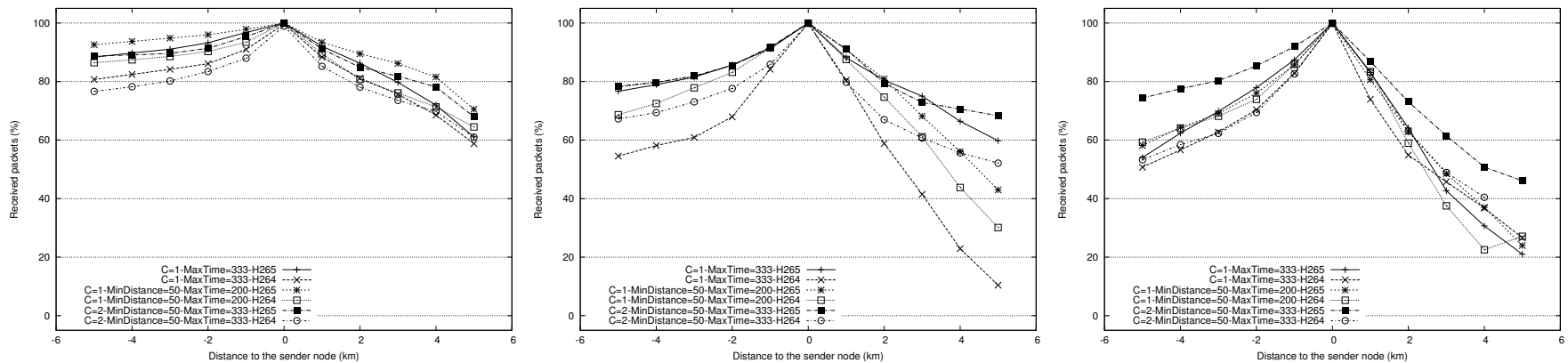

Figure 5. Packet delivery ratio. Vehicle arrival rate of $1 \mathrm{~s}$ (left), $2 \mathrm{~s}$ (center), $3 \mathrm{~s}$ (right).

analyzed the packets loss patterns, detecting differences in terms of the frame types affected. In particular, more I-frame losses occur in the forward transmission, being the I-frames type crucial to achieve a proper decoding of the video, while the P-frames and B-frames (frames that encode differences between the current frame and the previous I-frames) are not so critical.

Figure 7 shows the PSNR for the decoded frames. Although in some cases H.264 performs better than H.265, we have to take into account the differences in the number of decoded frames, since H.264 is only able to decode frames with a reduced packet loss, while H.265 is usually able to decode the entire video sequence. This explains why the H.264 decoded frames have better quality than the H.265 decoded ones, on average.

When both decoders have similar frame loss ratios, H.265 performs slightly better than H.264 in terms of PSNR.

These graphs highlight that H.265 outperforms H.264 in terms of both frame loss and PSNR quality. From the user perspective, the video flooded using the H.265 codec is able to provide a much smoother experience than H.264 encoded videos.

For informative purposes, some samples of the decoded videos are made available for download ${ }^{1}$.

\section{CONCLUSIONS AND FUTURE WORK}

In this paper we evaluated the user-perceived quality of real-time V2V video flooding in highway scenarios providing frame loss and PSNR data for the widely used H.264 codec and for the new H.265 video codec.

In our experiments we compared two flooding algorithms and selected the best configurations for each of the codecs

\footnotetext{
${ }^{1}$ http://www.grc.upv.es/WCNC-2014/videos.html
} 

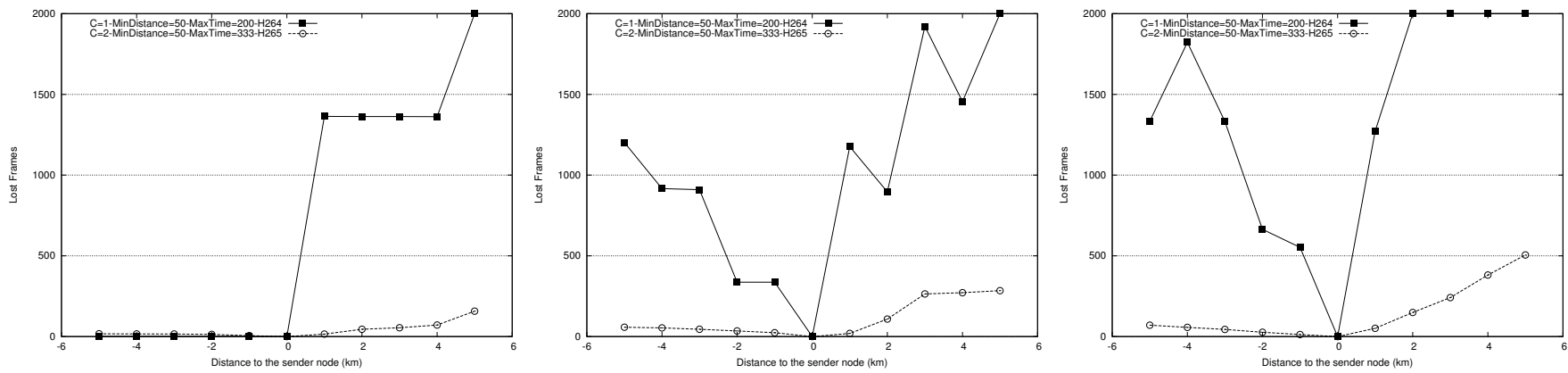

Figure 6. Frame loss. Vehicle arrival rate of 1s (left), 2s (center), 3s (right).
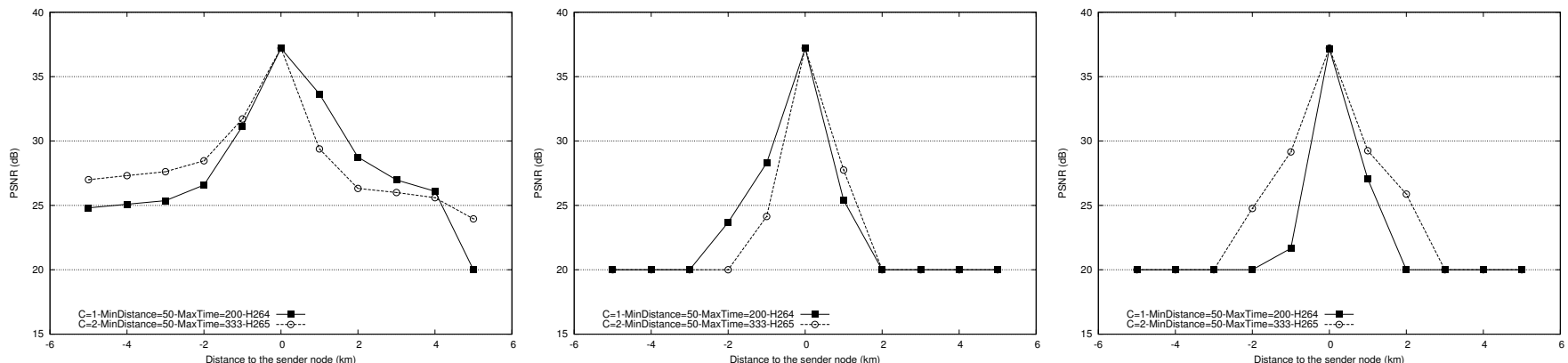

Figure 7. PSNR. Vehicle arrival rate of $1 \mathrm{~s}$ (left), $2 \mathrm{~s}$ (center), $3 \mathrm{~s}$ (right).

in terms of packet loss. It is noticeable that, due to the high level of medium congestion produced by the video stream, the better flooding algorithms are not the most aggressive ones, but those that make a more clever use of the medium.

After selecting the best configurations, a detailed analysis in terms of frame loss and PSNR for both of the codecs and for both highway directions is provided.

H.265 has shown to perform better than the H.264 codec, being more robust with high packet loss. Additionally, H.265 is able to maintain a controlled frame loss in all the situations, while H.264 is unable to provide an adequate frame rate to achieve a good user experience. In terms of PSNR for the decoded frames, both compression algorithms are unable to sustain the PSNR over $30 d B$ for distances greater than $1 \mathrm{~km}$, but the H.265 codec is able to provide a better visual quality than H.264 for a comparable frame loss rate.

Reducing the packet loss in general, especially losses associated with key-frames to avoid massive frame loss, is a key factor to achieve a good user experience. So, as a future work, we plan to design a new flooding algorithm that takes all these factors into account.

\section{ACKNOWLEDGMENTS}

This work was partially supported by the Ministerio de Economía y Competitividad, Spain, under Grant TIN201127543-C03-01, and by the Ministerio de Educación, Spain, under the FPU program, AP2009-2415.

\section{REFERENCES}

[1] "H.265 standard." http://www.itu.int/rec/T-REC-H.265.

[2] "H.264 standard." http://www.itu.int/rec/T-REC-H.264.

[3] H. Koumaras, M. Kourtis, and D. Martakos, "Benchmarking the encoding efficiency of h.265/hevc and h.264/avc," in Future Network Mobile Summit (FutureNetw), 2012, pp. 1-7, 2012.
[4] S.-Y. Ni, Y.-C. Tseng, Y.-S. Chen, and J.-P. Sheu, "The broadcast storm problem in a mobile ad hoc network," in Proceedings of the 5th Annual ACM/IEEE International Conference on Mobile Computing and Networking, MobiCom '99, (New York, NY, USA), pp. 151-162, ACM, 1999.

[5] Y.-C. Tseng, S.-Y. Ni, and E.-Y. Shih, "Adaptive approaches to relieving broadcast storms in a wireless multihop mobile ad hoc network," Computers, IEEE Transactions on, vol. 52, pp. 545 - 557, may 2003.

[6] F. Martinez, M. Fogue, M. Coll, J.-C. Cano, C. Calafate, and P. Manzoni, "Evaluating the impact of a novel warning message dissemination scheme for vanets using real city maps," in NETWORKING 2010 (M. Crovella, L. Feeney, D. Rubenstein, and S. Raghavan, eds.), vol. 6091 of Lecture Notes in Computer Science, pp. 265-276, Springer Berlin Heidelberg, 2010.

[7] M. Guo, M. Ammar, and E. Zegura, "V3: a vehicle-to-vehicle live video streaming architecture," in Pervasive Computing and Communications, 2005. PerCom 2005. Third IEEE International Conference on, pp. 171$180,2005$.

[8] F. Soldo, C. Casetti, C. Chiasserini, and P. Chaparro, "Video streaming distribution in vanets," Parallel and Distributed Systems, IEEE Transactions on, vol. 22, no. 7, pp. 1085-1091, 2011.

[9] P. Piñol, O. López, M. Martínez, J. Oliver, and M. P. Malumbres, "Modeling video streaming over vanets," in Proceedings of the 7th ACM workshop on Performance monitoring and measurement of heterogeneous wireless and wired networks, PM2HW2N '12, (New York, NY, USA), pp. 7-14, ACM, 2012.

[10] P. Seeling and M. Reisslein, "Video transport evaluation with h.264 video traces," Communications Surveys Tutorials, IEEE, vol. 14, no. 4, pp. 1142-1165, 2012.

[11] M. Behrisch, L. Bieker, J. Erdmann, and D. Krajzewicz, "Sumo simulation of urban mobility: An overview," in SIMUL 2011, The Third International Conference on Advances in System Simulation, (Barcelona, Spain), pp. 63-68, October 2011.

[12] "OMNeT++ simulator." http://www.omnetpp.org/. Acessed: April 4 2013.

[13] "INET framework." http://inet.omnetpp.org/. Acessed: April 4, 2013.

[14] M. Baguena, C. Calafate, J. Cano, and P. Manzoni, "Towards realistic vehicular network simulation models," in Wireless Days (WD), 2012 IFIP, pp. 1-3, 2012.

[15] "Video Traces." http://trace.eas.asu.edu. Acessed: January 15, 2013. 